\title{
ASERTIVIDAD E IDEOLOGÍA DE GÉNERO EN MUJERES VÍCTIMAS DE ABUSO PSICOLÓGICO
}

\section{Assertiveness and gender ideology in female victims of psychological abuse}

\section{Patricia Liranzo Soto ${ }^{1}$ y Libia Moreno Pérez}

Recibido: 8-5-2016 • Aprobado: 20-11-2016

\section{Resumen $^{1}$}

El propósito de esta investigación consiste en determinar la relación entre el nivel de asertividad y la ideología de género en mujeres víctimas de abuso psicológico. Igualmente, realizar intervenciones terapéuticas y educativas, a fin de promover cambios comportamentales que eviten que estas mujeres sigan siendo objeto de ese tipo de abuso. Este primer artículo comprende la fundamentación teórica donde se describe, se conceptualiza y se explica el abuso psicológico en sus dos tipos, manifiesto y sutil, enmarcado más ampliamente en el fenómeno de la violencia de género. Además, se presentan los conceptos principales y las aportaciones sobre las ideologías de género, tanto la tradicional como la igualitaria, considerando los planteamientos de la teoría del sexismo ambivalente de Glick y Fiske (Moya, Expósito \& Padilla, 2006) como una de las expresiones de la ideología tradicional de género. Del mismo modo, se desarrolla el concepto de asertividad, incluyendo su relación con la etnopsicología y la cultura. Para finalizar, se incluyen las reflexiones teóricas sobre el entrenamiento asertivo como una herramienta para que estas mujeres modifiquen sus creencias y puedan actuar con dominio en todos los ámbitos de sus vidas. Este marco conceptual ha servido como fundamento para la ejecución de los Talleres de Intervención Clínica y Psicoeducativa (TICPSI).

Palabras clave: psicología clínica; problemas emocionales; terapia de grupo; género; método psicoeducativo.

1. Profesora del Área de Ciencias Sociales del Instituto Tecnológico de Santo Domingo (INTEC). Correo electrónico: patricia. liranzo@intec.edu.do

\begin{abstract}
The purpose of this research is to determine the relationship between the level of assertiveness and gender ideology in female victims of psychological abuse. Also, conduct therapeutic and educational interventions, in order to promote behavioral changes that prevent these women from continuing to be subjected to psychological abuse. This first article includes the theoretical basis where psychological abuse is described, conceptualized and explained in its two types, overt and subtle, framed more broadly in the phenomenon of gender violence. In addition, the main concepts and contributions on gender ideologies, both traditional and egalitarian, are presented, considering the ambivalent sexism theory of Glick y Fiske (Moya, Expósito \& Padilla, 2006) as one of the expressions of traditional gender ideology. Similarly, the concept of assertiveness is developed, including its relationship with ethnopsychology and culture. Finally, the theoretical reflections on assertive training are included as a tool for these women to modify their beliefs and to act with dominion in all areas of their lives. This conceptual framework has served as the basis for the implementation clinical intervention and psychoeducational workshops.
\end{abstract}

Keywords: clinical psychology; emotional problems; group therapy; gender; psychoeducational method. 


\section{Introducción}

El propósito de este artículo es desarrollar el marco conceptual que fundamenta la comprensión de la ideología de género y la asertividad en mujeres que han experimentado abuso psicológico, lo cual sirve como base para los Talleres Clínicos y Psicoeducativos, así como para los dos artículos relacionados al tema en cuestión y que serán publicados posteriormente.

Los Talleres de Intervención Clínica y Psicoeducativos TICPSI fueron espacios exploratorios, vivenciales y reflexivos para constatar la manifestación de las ideologías de género y el bajo nivel de asertividad en las participantes. Además, fueron talleres de intervención para la modificación de las creencias estereotipadas con relación al género y el entrenamiento en habilidades asertivas, con el fin de que las participantes incorporaran herramientas para evitar el abuso psicológico al que estaban siendo sometidas. Dichos talleres se basaron en técnicas psicodramáticas y técnicas cognitivas conductuales.

Es importante señalar que, históricamente, las relaciones personales y sociales entre el hombre y la mujer han estado caracterizadas por la asimetría y la desigualdad. En la actualidad, muchas sociedades continúan siendo androcéntricas y colocan a la mujer en un segundo lugar, diferenciado y valorado como menor, tanto en términos de la participación y la actuación social, como en la vida privada y familiar. Una de las maneras en que el hombre ejerce dominio sobre la mujer es por medio del establecimiento de una relación de sometimiento emocional o abuso psicológico.

El abuso psicológico del hombre hacia la mujer se manifiesta por múltiples conductas, entre las que se encuentran: insultos, ofensas, humillaciones, críticas, desvalorización, entre otras. Esta forma de abuso se incluye dentro de la tipología de abuso contra la mujer, siendo los demás: abuso físico, sexual y económico. A pesar de ser un fenómeno antiguo y recurrente, este ha sido poco reconocido, identificado, descrito, denunciado e intervenido; su presencia se ha minimizado e ignorado, subestimando así las consecuencias dañinas en la psique femenina y en las relaciones de pareja.

Este tipo de abuso tiene entidad propia, por cuanto se manifiesta de manera independiente de la agresión física y puede llegar a percibirse como algo natural en las relaciones de pareja, justificándose por las conductas de género típicamente masculinas. De ahí que se crea, como piensan muchos autores, que las ideologías de género desiguales, con patrones de dominancia-control-poder en el hombre y sumisión-dependencia en la mujer, influyen y provocan las expresiones y las conductas abusivas del hombre hacia esta.

En adición a lo dicho, el estilo de paternidad predominante en la cultura dominicana tiende a ser el autoritario, exigiendo obediencia a sus hijos e hijas y limitando la expresión abierta de sus necesidades, deseos y opiniones; fomentando mayor pasividad en las niñas y estimulando conductas agresivas en los nińos. Es decir, no se educa en la asertividad, siendo una habilidad social necesaria para la defensa de los derechos propios y para establecer relaciones sanas y armónicas consigo mismo y con los demás.

\section{Objetivo general}

Determinar la relación que existe entre la asertividad y la ideología de género en mujeres víctimas de abuso psicológico, con el fin de realizar intervenciones educativas y terapéuticas, ofreciendo recursos y herramientas con la intención de promover cambios comportamentales que les impidan a estas mujeres ser o seguir siendo objeto de violencia de género. 


\section{Objetivos específicos}

- Evaluar la presencia de abuso psicológico en determinadas mujeres.

- Conocer el nivel de asertividad de mujeres víctimas de abuso psicológico.

- Identificar la ideología de género presente en las mujeres víctimas de abuso psicológico.

- Facilitar recursos educativos y terapéuticos a través de talleres de intervención para mejorar la asertividad y modificar las creencias tradicionales de las participantes.

- Evaluar los efectos de la intervención clínica y educativa en los temas de asertividad e ideología de género en las mujeres víctimas de abuso psicológico.

\section{Violencia y abuso psicológico}

Según la Organización Mundial de la Salud (OMS), la violencia se define como "el uso deliberado de la fuerza o el poder ya sea en grado de amenaza o efectivo, contra uno mismo, otra persona o un grupo o comunidad, que cause o tenga muchas posibilidades de causar lesiones, muerte, daño psicológico, trastornos del desarrollo o privaciones, y atenta contra el derecho a la salud y la vida de la población" (Horno, 2009, p. 15).

La violencia puede ser analizada desde el enfoque socioecológico de Brofenbernner, el cual explica que la persona es influida por diversos sistemas o niveles. En cada nivel existen factores protectores y factores de riesgo, estos últimos influyen en la violencia. En el ámbito individual, los factores de riesgo pueden ser la baja autoestima, el déficit de asertividad, la introversión, las pobres habilidades sociales y la dificultad para la resolución de conflictos. En el ámbito familiar, los factores de riesgo son una familia monoparental, una madre joven, los vínculos afectivos inseguros, el abandono, la familia numerosa, la drogodependencia o los trastornos físicos y psicológicos de algún miembro, etc. (Horno, 2009).

La mujer, como sujeto de acción dentro de las sociedades, ha tenido que luchar arduamente para constituirse como una ciudadana plena, con derechos igualitarios a los que posee el hombre. Como individuo con múltiples roles se ha manejado en ese transitar entre lo privado y lo público, queriéndose constreñir su accionar y su impacto como una actora clave en los procesos sociales. Estas circunstancias de cambio en la propia visión que asume la mujer postmoderna en su identidad personal y colectiva han generado múltiples consecuencias positivas y negativas. Su nueva actuación en los diferentes contextos ha hecho evidente la violencia a la que ha sido sometida ancestralmente.

La Organización Mundial de la Salud explica la violencia contra la mujer como:

todo acto de violencia de género que resulte, o pueda tener como resultado un daño físico, sexual o psicológico para la mujer, inclusive las amenazas de tales actos, la coacción o la privación arbitraria de libertad, tanto si se producen en la vida pública como en la privada (2013, para. 11).

La violencia contra la mujer se clasifica como física, psicológica y sexual. La violencia psicológica se entiende como las acciones que provoquen el desprecio al valor y a la dignidad personal, tratos humillantes, aislamiento, celotipia, amenazas, abandono y acciones que lleven al descenso de la autoestima y a dañar su desarrollo emocional (Ministerio de Salud Pública, 2011).

El Centro de Desarrollo Social y Asuntos Humanitarios de la ONU considera como violencia emocional: "los constantes malos tratos verbales, amenazas, hostigamiento, necesidad de hacer evidente la posesión sobre la persona, aislamiento de amistades y familiares, denegación de recursos 
físicos o económicos, dificultad al acceso a la educación, degradación, humillación en privado o en público y destrucción de objetos o animales favoritos" (Ministerio de la Mujer, (s.f.), p. 17).

En el marco de esta investigación se acogen los planteamientos de Marshall (Buesa \& Calvete, 2011), que incluyen la perspectiva de la relación dominancia-control en la construcción de lo que significa el abuso psicológico manifiesto y sutil. En ese sentido, se habla de abuso psicológico cuando existe el objetivo de sometimiento y control de la pareja. Dicho control se expresa en lo ya explicitado como violencia emocional, además de las conductas de menosprecio, crítica, irrespeto e ignorancia, así como la demanda de la obediencia, la negativa de colaboración, la retirada del afecto, el control del tiempo en el trabajo o ámbito educativo, en las relaciones personales y sociales, como las amistades, al igual que en la administración de las finanzas.

Marshall postula que estos intercambios dañinos pueden ser efectuados de manera explícita, pero también de manera sutil, por medio de "palabras específicas, como a través del tono de voz, expresiones faciales, gestos, etc., de manera que puede que no sea reconocido como abusivo por un observador o por la propia persona a la que va destinado el mensaje" (Buesa \& Calvete, 2011, p. 775). De esta forma, el conocimiento íntimo que tiene un individuo sobre la mujer, con respecto a sus fragilidades y aspectos negativos, así como de las situaciones en las que ella es vulnerable, se traduce en oportunidades para ejecutar el control de una manera implícita, lo que trae como consecuencia afectación en la relación amorosa.

El abuso psicológico sutil es un acto de violencia que debe ser comprendida desde dos conceptos: daño y poder. El primero es fácil de entender, no así el segundo. En todas las relaciones se manifiesta el efecto del poder en cuanto cada uno de los miembros tiene la capacidad de incidir sobre el otro; si se hace negativamente, propiciando un daño en el otro, se llega a la violencia. El amor es una forma de adquirir poder, como también lo es la autoridad, la fuerza o las diferencias. Para que el agresor ejerza violencia en la pareja, él abusa del poder ya que está cerca de la víctima, esta lo ama y ha incrementado su dependencia y su vulnerabilidad (Horno, 2009).

Marshall plantea distintas divisiones en torno al abuso psicológico manifiesto y sutil. Por su parte, el abuso psicológico manifiesto se clasifica en dominación y control; mientras que el abuso psicológico sutil se divide en clases como la desvalorización, la infravaloración y el aislamiento.

\section{Abuso psicológico manifiesto}

Dominación: esta categoría implica comportamientos agresivos o molestos y culpabilización, por parte de la pareja, lastimando los sentimientos de la otra persona cuando no se cumplen las exigencias.

Control: son conductas específicas que manifiesta la pareja, tales como espiar y no creer en lo que dice la otra persona, lo que puede llevar a esta última a mantenerse apartada de sus familiares y amistades.

\section{Abuso psicológico sutil}

Desvalorización: la pareja hace que la persona se sienta preocupada y/o insegura sobre su estado emocional y/o físico.

Infravalorización: se define como la conducta de lastimar el orgullo y el respeto que tiene la persona por sí misma, menospreciando las cosas que hace, manteniéndola apartada o actuando como si algo en ella estuviese mal, tanto en lo emocional como en lo mental.

Aislamiento: es cuando el agresor aparta a su pareja de las demás personas e incluso del tiempo que tiene esta para consigo misma, dificultando que vaya a alguna parte o hable con alguien más que no sea 
la pareja, pues es el único individuo que la comprende (O’Leary \& Maiuro, 2001).

Los vínculos afectivos se establecen a temprana edad en las interacciones de los padres y madres con sus hijos e hijas. Cuando las figuras parentales satisfacen las necesidades de sus hijos, estos muestran mayor independencia, seguridad y confianza en sí mismos. En cambio, cuando hay negligencia, abandono, maltrato, indiferencia y ambivalencia en el cuidado parental se crean vínculos inseguros en niños y niñas, caracterizados por conductas solitarias, poco sociables, autosuficientes, conductas negativas para llamar la atención o comportamiento desorganizado donde no pueden anticipar la conducta de las figuras parentales ni organizar la propia, formando así trastornos en su desarrollo (Feldman, 2007).

Hay estudios que refieren la continuidad del apego o vínculo. De esta manera, las relaciones románticas en la adultez con frecuencia repetirán los estilos de apego de la infancia y las parejas podrán mostrar apegos seguros que favorecerán el crecimiento y el bienestar mutuos, o vínculos inseguros que propiciarán relaciones destructivas, de maltrato, desapego y falta de compromiso (Liranzo \& Moreno, 2011).

Cuando la violencia ocurre a temprana edad y está relacionada con las figuras parentales, los efectos son mayores que cuando la violencia es presenciada o recibida de otras personas no significativas, ya que afecta su desarrollo afectivo y, en consecuencia, resultan perjudicadas las demás áreas. Los niños y las niñas maltratados por sus padres y madres aprenden a asociar como una norma los factores amor-violencia (Horno, 2009).

Horno (2009) plantea que existen varias razones por las que las víctimas prefieren una relación maltratante a no tener dicha relación. Por un lado, las fases del ciclo de la violencia activan sus mecanismos de disonancia cognitiva para justificar las agresiones recibidas; por otro lado, eligen permanecer en una mala relación, pero en la que poseen parte de su identidad social, al sentir que no pertenecen a nada, por lo que si no tienen otras opciones de reestructuración de su vida mantienen la relación abusiva.

Una mujer víctima de maltrato emocional está acostumbrada al irrespeto, a la falta de consideración, la rudeza, la indiferencia y puede experimentar este intercambio como algo natural, debido a que usualmente estas víctimas provienen de familias con historias de algún tipo de maltrato o abuso (Romero, 2012).

El síndrome de Adaptación Paradójica a la Violencia Doméstica se define como un conjunto de procesos psicológicos, cognitivos, emocionales y conductuales que culmina en la formación de un vínculo interpersonal de protección entre la víctima y el agresor en el contexto de un ambiente traumático. Se percibe como un mecanismo activo de adaptación habilitado para amortiguar y/o evitar la incidencia masiva de estresores amenazantes sobre la víctima (Montero, 2001).

La experiencia de maltrato continuo, en cualquiera de sus formas, incrementa el daño psíquico en la mujer, produciendo en ella afectación psicológica y dificultad en su funcionamiento global. Dentro de los síntomas experimentados que reflejan el daño psíquico se encuentran: "baja autoestima, síntomas depresivos, estrés y alteraciones somáticas, sensación de abandono, impotencia, sentimientos de culpabilidad y labilidad afectiva" (Cartagena, Barrero \& Donat, 2012, pp. 193-194).

Diversos estudios se han realizado con respecto al tema del abuso psicológico y su asociación con otras tipologías de abuso u otras variables. Al respecto, Culay, Santana, Rodríguez y Pérez (2000), en su investigación Mujer y violencia: un problema de salud comunitario, con una muestra de 1,088 mujeres, encontraron que el $73 \%$ reportó algún tipo de maltrato conyugal, siendo el psicológico el más frecuente en $50 \%$ de ellas, con la acotación 
de que la gran mayoría no había solicitado ayuda profesional.

La investigación Maltrato físico y psicológico en mujeres victimas de violencia en el hogar, realizada por Amor y Echeburría (2001), permitió constatar que el 38\% de la muestra que asistió al Centro de Asistencia Psicológica para Víctimas de Violencia fue por abuso psicológico y la búsqueda de ayuda se hizo luego de permanecer 10 años en una relación abusiva. Estos autores destacan que, aunque las víctimas de abuso psicológico se encuentran en menor gravedad, tienen intenso malestar emocional al igual que las víctimas de violencia física, evidenciándose una divergencia entre la gravedad objetiva y la subjetiva. Si bien la violencia psicológica puede ser el escalón para llegar a la violencia física, en muchos casos es una entidad en sí misma, sin pasar los límites a otro tipo de violencia.

Vázquez, Torres y Otero (2010), en el estudio Prevalencia y factores de riesgo de la violencia contra la mujer en estudiantes universitarias españolas, encontraron que el $15 \%$ de las estudiantes había recibido algún tipo de maltrato. El abuso más frecuente fue el psicológico en el 65\% y los factores asociados fueron un nivel cultural medio, vivir en zona urbana y haber presenciado situaciones de abuso en la infancia.

A través del estudio Prevalencia de casos de violencia familiar contra la mujer en las etapas de embarazo, parto y puerperio, realizado en la Universidad de Buenos Aires por Colombo, Ynoub, Viglizzo, Beneranda, Iglesias y Stropparo (2005), se encontró que el $28 \%$ padecía violencia. Mientras que $52 \%$ fue víctima de violencia física y psicológica: el $35 \%$ de violencia psicológica y el $12 \%$ sufrió los tres tipos de violencia.

En la investigación de Calle (2004), denominada Consideraciones sobre la victimización secundaria en la atención social a las víctimas de violencia de género, se describe que los sistemas sanitario, social, policial, judicial y educativo pueden reproducir situaciones de violencia con acciones que contribuyen a la ocultación, minimización de la violencia o derivación precipitada a otros servicios. A su vez, explican que no existen tratamientos estándar, sino que hay que individualizar el proceso de atención enfocándose en la historia, la personalidad, las creencias y el momento o la etapa del ciclo de violencia en que se encuentra la víctima.

\section{Ideología y rol de género}

La identidad del ser humano se construye a lo largo de todo su ciclo vital e incluye aspectos biológicos y aspectos adquiridos. Dentro de los primeros aspectos se encuentra el sexo, que se manifiesta por la estructura anatómica y fisiológica que muestra diferencias entre hombre y mujer $y$, aunque se nace con este sexo biológico, las personas se desarrollan psicológicamente en un proceso que lleva a la construcción individual activa de aspectos tales como: la sexualidad, la identidad de género, el rol de género y la orientación sexo-erótica. Se denomina rol de género a la manifestación pública de la identidad interiorizada a través de la ejecución de diversos papeles en la vida sexual: padre, madre, esposo, esposa, amigo y amiga (González \& Castellano, 2003).

Los roles de género se describen como los preceptos vinculados o las condiciones idóneas para lograr el estatus de hombre o mujer. En los actos de violencia hacia la mujer se evidencia un esquema masculino caracterizado por la rigidez y la malicia en contraposición a las cualidades femeninas que son vulnerabilidad, debilidad, necesidad de protección y control. Todas estas cualidades parten de creencias que son transferidas como normas de conductas anheladas que se interiorizan en la propia identidad del individuo, la cual se recrea desde el dominio externo de comportamiento al dominio interno que se reproduce en las ideologías de los géneros (Delgado-Álvarez, Sánchez-Gómez \& FernándezDávila, 2012). 
Estas mismas autoras plantean que:

Los roles de género aparecen estrechamente ligados al concepto de "estereotipo de género", concebido como un proceso de construcción psíquica que engloba diversos atributos, que tiene un carácter funcional y evaluativo, con posibilidad de cambio. Aunque en principio el concepto estereotipo supone una valoración neutra, más bien se relaciona al concepto de prejuicio cuando se aplica a grupos con menor poder social. (p. 771)

La cultura patriarcal ha configurado rígidos estereotipos de lo masculino y femenino que se expresan en las variadas conductas del individuo, desde los juegos, los vestuarios, la forma de comportarse, de comunicarse y de expresar los sentimientos; hasta las aspiraciones y proyecciones educativas, profesionales y laborales (González \& Castellano, 2003).

Estos estereotipos se les transmiten a los niños y las niñas en el proceso de socialización en sus diferentes ambientes familiares, escolares y comunitarios; a su vez, se convierten en lineamientos culturales de tal manera que se interiorizan como naturales y universales y se ejecutan para obtener el reconocimiento social, incluso a costa de anular sus necesidades, intereses y aspiraciones íntimas (González \& Castellano, 2003).

El género tiene un papel relevante en la conformación de la identidad personal en el contexto social, por lo que se espera que en la interrelación entre lo femenino y lo masculino existan estereotipos mutuos y que los mismos tengan un peso considerable y sean de una gran complejidad. En contraste, muchas culturas ven a la mujer como una persona más maleable, sumisa, expresiva de sus sentimientos, propensa a los cambios en su estado de ánimo y más frágil frente al estatus del hombre (Morales $\&$ López-Sáez, 1996).

El constructo ideología de género ha sido usado para definir las ideas que los sujetos poseen acerca de los roles y las conductas que hombres y mujeres deberían realizar y de las interacciones entre ellos y ellas, diferenciándose del concepto estereotipos de género que implica los pensamientos acerca de las diferencias en los atributos personales de hombres y mujeres. Actualmente, se usa el constructo ideología de género o sexismo (Moya, Expósito \& Padilla, 2006).

Otro concepto de ideología de género es el que plantean Davis \& Greenstein (2009), quienes lo explican como la idea subyacente a nivel personal que se tiene sobre la división del trabajo y de las responsabilidades familiares entre hombres y mujeres, fundamentada en la noción de que son esferas separadas.

La ideología de género se concibe como una dimensión cuyos extremos podría definirse en dos polos: la ideología tradicional de género y la ideología igualitaria de género. El primer polo enfatiza las diferencias entre los sexos considerando a las mujeres como débiles y necesitadas de protección, mientras que los hombres ejercen la autoridad, tienen funciones de cuidado y son proveedores, ocupando lugares en la esfera pública y externa al hogar.

En los hombres que se guían por una ideología tradicional se evidencian limitaciones en la expresión emocional, conductas violentas, así como conductas sexuales de riesgo. En las mujeres guiadas por esta ideología se perciben menos aspiraciones laborales y poca importancia a su carrera profesional. En el segundo polo, la ideología igualitaria expresa que las diferencias de género son de carácter social y establece que debe haber igualdad en los roles, las tareas y las funciones entre ambos sexos (Moya, Expósito \& Padilla, 2006).

Bolzendahl y Myers argumentan que las actitudes hacia los asuntos feministas incluyen el concepto de la ideología en función de las explicaciones basadas en el interés o en la exposición. De esa manera, las descripciones fundamentadas en el interés recaen en la estructura del individuo, es decir, en sus metas personales. Cuando las personas se benefician de 
la igualdad de género son más propensas a asumir creencias igualitarias (Davis \& Greenstein, 2009).

Barnette y Rivers indican que la mayoría de las personas no mantendría creencias igualitarias, debido a que los intereses en la estructura de la mujer y del hombre presentan expectativas culturalmente diferentes, fundamentadas en las creencias hegemónicas que ratifican la noción polarizada de las diferencias de género, las cuales se convierten en realidad. Esto ilustra por qué las mujeres tienen una ideología de género más igualitaria que los hombres, debido a que los hombres se inclinan en menor medida a creer que la igualdad de género los favorecería (Davis \& Greenstein, 2009).

Las explicaciones basadas en la exposición, según Bolzendahl y Myers, describen que estar frente a ideas o circunstancias que son consonantes con los ideales igualitarios promueve el desarrollo de creencias más igualitarias. Esta exposición se hace evidente a través de la socialización, la educación y la experiencia personal (Davis \& Greenstein, 2009).

La ideología del padre y de la madre se asocia positivamente con las ideologías de género del niño y de la nińa, de tal manera que padres y madres más igualitarios pueden tener infantes con una visión de género más igualitaria. Sin embargo, independientemente de la socialización, la prominencia de los efectos de la familia de origen en la formación de actitudes parece retroceder durante la adolescencia, ya que la influencia de los compañeros y las compañeras, al igual que de sus propias experiencias de vida se vuelve más fuerte (Davis \& Greenstein, 2009).

La educación provee exposición a ideas igualitarias y contrarresta la aceptación de mitos y estereotipos de género. La investigación de panel realizada por Trendand ha demostrado que el aumento de la educación se asocia con un mayor igualitarismo de género. De hecho, Bryant informó que tanto las mujeres como los hombres se vuelven menos tradicionales después de cuatro años de universidad y las experiencias universitarias que conducen a este cambio son similares para hombres y mujeres (Davis \& Greenstein, 2009).

Una consideración a destacar es que las personas pudieran tener una tendencia menos igualitaria, como manera de reducir la disonancia cognoscitiva que experimentan en la interacción personal, donde se espera una ideología igualitaria, pero se vive la inequidad de género como una norma históricamente dada, situación que se evidencia en el matrimonio y como padres y madres (Davis $\&$ Greenstein, 2009).

Hernández y Pacheco (2009b) explican que el patriarcado define una idea del ser mujer y este ser mujer está sujeto a la estructura de lo masculino. Postulando a Pisano (2001), estas autoras llaman la atención sobre cómo lo masculino requiere de mujeres como ayudantes, subalternas, colaboradoras, etc.; mujeres que se circunscriban a un contexto cultural y enfoquen su accionar alrededor de las creencias y las expresiones de lo masculino.

Hernández y Pacheco (2009) recreando lo planteado por Lagarde (2003) señalan que, los estereotipos de género conferidos a las mujeres y la dificultad que tienen estas de llevar a cabo dichas obligaciones en sus diversos y variados roles: madre, ama de casa, esposa y amante, suscitan en ellas tres expresiones de la culpa: creerse mujeres incompletas, tener una identidad estereotipada y sentirse poco competentes ante las demandas sociales.

En ese mismo tenor, Esiler apunta: "Las mujeres a veces soportan relaciones abusivas porque han sido eficazmente condicionadas a culparse a sí mismas por su sufrimiento y tienen la esperanza que si de alguna manera logran cambiar para no enfurecer a sus abusadores, todo funcionará bien" (Esiler, 1998, citado por Hernández \& Pacheco, 2009b, p. 509).

Expósito, Moza y Glick explican que los estereotipos de género llevan a la discriminación de género, la que se define como los actos desiguales hacia personas que son de un determinado sexo y que tienen 
un efecto dañino sobre ellas. Esto se ha llamado también sexismo (Aranda \& Montes, 2012).

Existen dos teorías acerca de la discriminación de género en la actualidad: el neosexismo y el sexismo ambivalente. La primera teoría evidencia una contradicción entre los valores igualitarios y los sentimientos negativos hacia las mujeres, de tal manera que disminuyen las conductas explícitas de discriminación, pero se mantienen los prejuicios contra ellas (Aranda \& Montes, 2012).

La segunda teoría refleja la coexistencia de dos tipos de sexismo: el hostil y el benévolo. El sexismo hostil es el tradicional que mantiene la idea de la inferioridad femenina. El benévolo, por su parte, muestra una actitud de respeto y protección hacia las mujeres, siempre que estas se mantengan en sus roles tradicionales; este es perjudicial porque es encubierto y, por tanto, es difícil de identificar (Aranda \& Montes, 2012).

Las mujeres son las receptoras de las conceptualizaciones culturales que disminuyen el ámbito de acción de ellas mismas. A pesar de los cambios acaecidos, aún continúa la adjudicación de lo privado como lo femenino y de lo público como lo masculino, barrera que imposibilita la garantía de derechos a la mujer, el acceso al poder y la toma decisiones, comportamientos sociales que se perpetúan por medio de los estereotipos de género (Pola, 2005).

A pesar de los innumerables esfuerzos realizados por las diferentes instituciones sociales, nacionales e internacionales, para fomentar cambios en la actitud y en el comportamiento frente a estos estereotipos de género, persisten en algunos sectores del imaginario social creencias acerca de la mujer que parecerían inmodificables, entre las que están: "las mujeres son inferiores a los hombres", "el hombre es el jefe del hogar", "el hombre tiene derechos de propiedad sobre la mujer y los hijos", "la privacidad del hogar debe ser defendida de las regulaciones externas" (Corsi, 2003, p. 20).
El estudio El impacto de los estereotipos y roles de género en México, realizado por el Instituto Nacional de las Mujeres (2007), permitió detectar que el 35\% de las participantes víctimas de violencia dicen que las mujeres deben obedecer a su esposo en lo que él ordene y el $68 \%$ dice que es el hombre quien debe responsabilizarse de los gastos familiares.

En consonancia con lo anterior, la proporción de mujeres que pide permiso o tiene en cuenta la opinión del esposo para tomar decisiones es grande. Una vasta mayoría de mujeres, independientemente que trabajen fuera del hogar o no, participan en mayor medida que los hombres en las labores domésticas. Es de resaltar que las mujeres jóvenes no casadas reprueban en su mayoría las actitudes de dominancia y poder de sus parejas y asumen actitudes de mayor libertad en la toma de decisiones y en el control de sus vidas.

De Lemus, Moya, Bukowki y Lupiańez (2008), en su estudio Activación automática de las dimensiones de competencia y sociabilidad en los casos de los estereotipos de género, detectaron que ante la presencia de imágenes de hombres y mujeres en estudiantes de Psicología, en condiciones experimentales que limitaban los recursos cognoscitivos y maximizaban el pensamiento automático, los y las participantes clasificaron la dimensión competencia asociada a los hombres y la de sociabilidad a las mujeres.

Otros estudios realizados con la escala de ideología de género en España y Latinoamérica han hecho posible constatar que el patrón general de las medias de las puntuaciones totales hacia el polo igualitario de la escala indica que las mujeres puntúan superior de manera significativa más que los hombres. También se obtuvieron puntuaciones menos igualitarias en muestras de mayor edad y menor nivel educativo (Moya, Expósito \& Padilla, 2006).

Hernández y Pacheco, en su investigación Nueva mirada psicológica al ser mujer, despertar, transgredir y nacer en el arte (2009a), encontraron que el ser mujer continúa siendo experimentado desde el 
dominio masculino, a pesar de que hubo visiones y propuestas críticas al respecto. Dentro de las condiciones que debe cumplir la mujer están la demanda de lucir bella y la imposibilidad de vivir sola, por lo cual debería vivir con la pareja o con los familiares, lo que fortalece la visión de que está incompleta y que solo podría llenarse a partir de ser para los otros.

Otro elemento relevante de los hallazgos fue que "el patriarcado configura identidades femeninas a partir de la negación y la demostración de las propias capacidades. Se trata de una combinación entre un no debes y debes demostrar que eres capaz" (p. 334). A su vez, hay una culpa, dicen las autoras, que dificulta que la mujer se haga responsable de su vida, lo que provoca una afectación importante en su autovalía, pues ella no piensa que es capaz o merecedora.

\section{Asertividad}

El primer grupo social donde un individuo se experimenta como ser humano es la familia y esta, junto con la sociedad, el contexto histórico y la cultura, modela formas de interrelación que permiten no solo el contacto entre las personas, sino el establecimiento de vínculos y relaciones sociales, ya sean superficiales o profundos. Dentro de esta relación se supone que las partes puedan expresarse de manera adecuada, pero no siempre ocurre así, por lo que la persona puede comportarse de manera variada, tanto con una conducta pasiva como con una conducta agresiva, cuando se identifica que el comportamiento idóneo descansa sobre el comportamiento asertivo.

La asertividad, habilidad asertiva o conducta asertiva tiene múltiples definiciones para conceptualizarla, debido al marco referencial desde donde sea entendida. El concepto asertividad proviene del latín assere o assertum que quiere decir defender o afirmar. Desde esta perspectiva, siguiendo a Rodríguez y Serralde, la asertividad pudiera significar la validación de la personalidad, la seguridad en sí mismo, la autoestima, la ecuanimidad y una comunicación convincente y eficaz (Rodríguez y Serralde, citado por Gaeta \& Galvanovskis, 2009).

Este concepto puede comprenderse desde varias aproximaciones teóricas. A partir del enfoque conductual, el aprendizaje asertivo se basa en el condicionamiento clásico de Iván Pavlov. De este modo, si en el individuo se manifiestan prominentemente los impulsos excitatorios, tenderán a ser personas proactivas, emocionalmente independientes, confrontativos y a vivir la vida desde su propia visión. En cambio, si los impulsos de mayor manifestación son inhibitorios, las personas propenderán a sentirse desorientadas, temerosas, reprimirán sus emociones y, frecuentemente, se mostrarán compelidas a hacer lo que no desean. El balance entre inhibición y excitación conllevará a comportamientos sumisos (pasivos) o agresivos (Gaeta \& Galvanovskis, 2009).

Desde la aproximación cognoscitiva, la asertividad es considerada como la forma de manifestar las creencias, los sentimientos y lo que se aspira, de una manera directa y honesta, en la asunción de los derechos propios y la consideración de los derechos de los otros. En ese sentido, Flores argumenta que el comportamiento asertivo se basa en la falta de ansiedad frente a los contextos sociales, en donde para la persona es posible expresar emociones, pensamientos y acciones (Flores, 1994, citado por Gaeta \& Galvanovskis, 2009).

El enfoque humanista incluye la asertividad como una estrategia en el proceso de desarrollo de la autorrealización. Picky \& Vargas postulan que "para ser asertivo se necesita aceptarse y valorarse, respetar a los demás, permanecer firme en las propias opiniones, comunicar con claridad y directamente, en el lugar y momento adecuados y de forma apropiada, lo que se quiere o necesita decir" (Picky \& Vargas, 1990, citado por Gaeta \& Galvanovskis, 
2009, p. 405). Zaldivar (2002) agrega que la conducta pasiva implica la falta de acción en la defensa de los propios derechos y la facilitación a que los otros manipulen y se beneficien de esta situación.

La asertividad también involucra acciones que posibilitan a la persona comportarse desde su propio interés, haciendo valer sus derechos sin vivenciar angustia y expresando la legitimidad de sus sentimientos de manera fluida y sin que estos vayan en detrimento de los sentimientos y derechos de los y las demás (Vatankhah, Daryabari, Ghadami \& Naderifar, 2013). En algunos individuos la asertividad se expresa de manera natural, pero es una habilidad que puede ser aprendida. Las personas que son asertivas en su interrelación con los y las demás son capaces de disminuir en gran medida los conflictos interpersonales, por lo tanto, reducen una gran fuente de estrés (Pourjali \& Zargnahash, 2010).

Rodríguez \& Serralde señalan que la persona asertiva admite sus limitaciones al asumir que no siempre puede obtener lo que quiere, acepta o no en su esfera emocional a otros individuos, pero lo hace de una forma cuidadosa y segura; establece quiénes son sus amistades y es capaz de expresar sus sentimientos (Rodríguez y Serralde, citado por Gaeta \& Galvanovskis, 2009). Por su parte, Castanyer (1996) explica:

El que una interacción nos resulte satisfactoria depende de que nos sintamos valorados y respetados y esto, a su vez, no depende tanto del otro, sino de que poseamos una serie de habilidades para responder correctamente y una serie de convicciones o esquemas mentales que nos hagan sentirnos bien con nosotros mismos (p. 20).

Esta misma autora indica que la asertividad es una vía hacia la autoestima, hacia la capacidad de los individuos de vincularse entre sí en el mismo grado, ni por encima, ni por debajo. A pesar de ello, la expresión de la asertividad puede ser bloqueada por el desconocimiento de los derechos asertivos, por ansiedad o por temor ante la ejecución de la expresión asertiva y por la presencia de creencias irracionales relacionadas con la necesidad de tener aprobación social en los diferentes contextos (Zaldívar, 2002).

Es importante destacar que las conductas asertivas no son de carácter innato, sino que se integran por aprendizaje a lo largo del desarrollo. En ese mismo orden, la educación juega un papel preponderante para fomentar comportamientos asertivos. Las habilidades sociales que intervienen en la conducta asertiva se logran por la experiencia, es decir, por el aprendizaje a través de la observación, la comprobación, la imitación y la información. Todas ellas aumentan el aseguramiento social que recibe la persona y que es matizada por la peculiaridad de las circunstancias, el grupo etario, el sexo y el estatus social (Velásquez, et al., 2008).

\subsection{Asertividad y cultura}

El comportamiento asertivo es de carácter aprendido y se encuentra circunscrito a un contexto social y cultural, lo que quiere decir que ciertas conductas individuales están matizadas por las normas sociales establecidas, que varían de una cultura a otra.

Hofstede argumenta que: "los individuos de culturas con alta distancia de poder, la cultura latinoamericana entre ellas, valoran la conformidad y obediencia, así como apoyan actitudes autocráticas y autoritarias de quienes están a cargo de organizaciones e instituciones (Hofstede, 1991, citado por Zubieta, Fernández, Vergara, Martínez \& Candia, 1998, p. 4).

Con respecto a la expresión de las emociones, uno de los factores del comportamiento asertivo, las culturas latinoamericanas se manejan con un derrotismo imbuido de religiosidad, el conformismo frente a la adversidad y la resignación ante el destino. Además, la admisión del sufrimiento en la vida, una autorregulación emocional firme, o sea, 
no emocionarse por los acontecimientos vitales y no experimentar intensas alegrías o tristezas, todas se relacionan a la distancia al poder (Zubieta et al., 1998).

En cuanto a la dimensión masculinidad-feminidad, Latinoamérica presenta una gran variación, pues hay culturas que se inclinan más a una feminidad social y otras a la masculinidad social. En este caso, un escenario común es el machismo y el marianismo. El machismo no solo se refiere a la superioridad del hombre sobre la mujer, sino que este hombre se asume y es percibido como alguien fuerte, que suscita respeto y cuyo deber es la protección de la familia. El marianismo se caracteriza por una visión de mujer resignada al sufrimiento y sometida a su familia, a su rol de pareja y a su destino. Esta gran diferenciación de los géneros, empero, no se manifiesta en todas las culturas de América Latina (Zubieta et al., 1998).

Otro aspecto de suma importancia es la evaluación positiva de la familia, cuya asunción es característica del colectivismo latino. Este nivel de importancia hacia la familia consolida los vínculos interdependientes, incluyendo una firme identificación con el respaldo, la reciprocidad y la fidelidad. El intercambio se hace con la familia cercana, la familia extendida y con las amistades a las que se le asigna un rol de cuasi parientes (Zubieta et al., 1998).

En el ámbito de las normas de expresividad en las culturas colectivistas como la latinoamericana, el hecho de manifestar sentimientos y afrontar la experiencia emocional está sujeto al ajuste de los roles contextuales y al manejo de la impresión que se causa, o sea, de lo que pueda percibir y valorar el otro (Zubieta et al., 1998).

En el marco de lo antes expuesto, la cultura es un elemento fundamental a considerar para la comprensión del ser humano; a esto se le llama Etnopsicología, que se define como "los efectos fundamentales y primordiales que una cultura tiene sobre la personalidad de los individuos que nacen y se desarrollan dentro de ella" (Díaz-Guerrero, 1972, citado por Alarcón, 2010, p. 15).

Este mismo autor, en sus investigaciones sobre la identidad del pueblo mexicano con respecto a sus actitudes, valores y afectividad, planteó que la sociocultura media las expresiones y las acciones psicológicas fundamentales en la vida de toda persona. A partir de la utilización de su Escala de Premisas Histórico y Socio Culturales, pudo conocer y establecer nueve factores del comportamiento de los sujetos.

Aunque Díaz-Guerrero hizo sus estudios en la población mexicana, algunos de los factores por él explicados guardan similitud con otras culturas latinas, como la dominicana, ya que comparten experiencias y semejanzas. Estos factores son el machismo, la obediencia afiliativa, la virginidad, la abnegación, el temor a la autoridad, el statu quo familiar, el respeto sobre el amor, el honor familiar y la rigidez familiar (Alarcón, 2010).

Con respecto al machismo, la mujer es percibida como obediente, sumisa, con menor capacidad que el hombre, inferior y debe ser protegida. La obediencia afiliativa significa un respeto total e incondicional al padre y a la madre; ambos deben ser amados y honrados. La abnegación implica que la mujer tolera y padece más el sufrimiento que el hombre, por lo cual es más sensible; entonces para ella, más que para el hombre, la vida hay que aguantarla.

El temor a la autoridad se trata de constatar qué tanto la persona expone su punto de vista fundamentado en su experiencia particular. Con respecto a la relación entre padres e hijos se evidencia el grado de temor que en la cultura del individuo tienen los hijos hacia los padres. El statu quo se refiere a la lealtad entre los padres, a cómo los hijos desean ser como su padre y su madre y a que las mujeres deben ser cuidadas y el honor de la familia ha de conservarse (Alarcón, 2010). Estos factores fueron vueltos a evaluar en 1994 y muchos de ellos 
disminuyeron, lo que valida la afirmación de DíazGuerrero de que los mandatos socioculturales se pueden modificar, aunque no al mismo ritmo.

Para la Etnopsicología, el desarrollo cognoscitivo y de personalidad se despliega a partir de una argumentación constante entre el ser humano bio-psíquico y su entorno sociocultural (Flores, 2010). El concepto de asertividad postulado por Flores es:

La habilidad verbal para expresar deseos, creencias, necesidades y opiniones, tanto positivas como negativas, así como también para establecer límites de manera directa, honesta y oportuna, respetándose a sí mismo como individuo y durante la interacción social, entendiéndose esta última como: a) las relaciones o situaciones de la vida cotidiana en las que existe una interacción con desconocidos; b) las relaciones afectivas en donde existe una interacción con personas involucradas sentimentalmente, es decir, familia, amigos y pareja; y c) las relaciones educativo-laborales en las que se interactúa con autoridades y compañeros en un contexto sociocultural determinado. De esta manera, se considera también de importancia el contexto en el que se desenvuelve la persona (Flores, 1994, citado por Gaeta \& Galvanovskis, 2009, pp. 410-411).

Flores y Díaz Loving explican que la capacidad de manifestar un comportamiento asertivo, en la mayoría de las situaciones, se ajusta al ámbito cultural en que la persona crezca. Aun en una misma cultura, existirán diferencias por el estatus social, la personalidad, el sexo y la edad (Flores, 2010).

\section{Entrenamiento asertivo}

Cuando a un individuo se le hace difícil mantener un comportamiento asertivo, esto le trae como consecuencia dificultad en la convivencia y en el mantenimiento de relaciones sociales saludables. A sabiendas de que se ha dicho que el comportamiento asertivo es mayormente aprendido, existen técnicas para poder subsanar estos déficits; al conjunto de estas técnicas se le denomina entrenamiento asertivo.

El entrenamiento asertivo se puede definir como una de las maneras para lidiar con los problemas que se hacen presente en el intercambio personal, los cuales están caracterizados por inconvenientes para la manifestación de las emociones con respecto, en primer lugar, a sí mismo y luego a los demás. Por medio del entrenamiento asertivo el individuo alcanza la habilidad para una expresión efectiva de sus puntos de vista, sus emociones y sus anhelos particulares, haciéndolo de una forma apropiada, verdadera y directa (Zaldívar, 1994).

El entrenamiento asertivo faculta a la persona para conducirse de manera apropiada frente a los siguientes elementos: angustia, miedo, culpabilidad, ideas distorsionadas, afirmaciones o creencias que de alguna manera cohíben la manifestación de la asertividad (Zaldívar, 1994).

El entrenamiento asertivo destaca la autoexpresión como una manera socialmente favorable que pudiese, por ejemplo, referirse a un aumento de la autoestima, una visión de inferioridad disminuida y un locus de control acrecentado. Su propósito es aumentar el repertorio comportamental por medio de un sinnúmero de situaciones, como parte del proceso interrelacional en el cual los seres humanos se sienten confortables tanto de sí como del otro (Castaños, Reyes, Rivera \& Díaz, 2011).

Este entrenamiento está sujeto a la comprensión e identificación de los matices del comportamiento asertivo, que son: el aspecto comportamental, cuáles son las conductas que las personas exhiben; el aspecto personal, quiénes son el objetivo de la manifestación de la conducta; $y$, por último, el aspecto situacional que se refiere al lugar donde se expresa 
el comportamiento asertivo, si es en el ámbito público o privado (Castanyer, 1996).

Castanyer (1996) indica que, para entrenar en un comportamiento asertivo, hay que tomar en cuenta los tres grados de funcionamiento en una conducta: el emocional, el cognoscitivo y el motórico. Generalmente, la persona puede exhibir problemas en más de un aspecto, por lo cual se comienza con el componente más sobresaliente. Sobre esta base, hay tres tipos de estrategias principales o conjunto de técnicas que son: la reestructuración cognitiva, el entrenamiento en habilidades sociales y la técnica de reducción de la ansiedad, a la cual se le añade la estrategia de resolución de problemas.

En el estudio de León, Rodríguez, Ferrel \& Ceballos (2009), titulado Asertividad y autoestima en estudiantes del primer semestre de la facultad de Ciencias de la Salud en una universidad pública de Santa Marta, se encontró que los estudiantes que presentaron comportamientos asertivos a su vez calificaban con una autoestima alta.

Con respecto a la familia, dichas mujeres conservan y sostienen la estructura tradicional de interrelación entre sus miembros, donde se privilegia la obediencia y el miedo de los hijos a los padres. En lo concerniente a la pareja, estas mujeres expresan dinámicas tradicionales, donde el hombre es quien dirige y la mujer acata, pudiendo protestar solo a través de una emocionalidad negativa. Además, se evidenció que las normas históricas-socio-culturales reflejan y fomentan la inequidad entre los géneros, contribuyendo a la perdurabilidad de la violencia contra la mujer.

En la investigación Bienestar psicológico, asertividad y rendimiento académico en estudiantes universitarios Sanmarquinos, realizada por Velásquez et al. (2008), se encontró que existe una relación positiva entre el bienestar psicológico y la asertividad.

Pourjali y Zarnaghash (2010) realizaron un estudio en Irán, que se tituló Relationships between assertiveness and the power of saying no with mental health among undergraduate students. Los hallazgos explican que existe una relación entre la asertividad y el poder de decir no con la salud mental de los estudiantes de la universidad de Shiraz. Tanto el comportamiento asertivo, como ejercer el derecho de decir no afecta de manera positiva el bienestar psicológico.

Cuando se presenta una alta conducta asertiva hay mayor conservación de la salud mental. De igual forma, cuando el individuo tiene el poder de decir no, experimenta menos estrés en su vida y es capaz de satisfacer sus necesidades, lo cual también significa menos estrés por las necesidades no satisfechas. Además, la persona es capaz de ayudar a los demás a cumplir con sus necesidades, lo que redunda en una mejor red de apoyo social.

El estudio Programas de intervención en mujeres victimas de violencia de pareja y con agresores: experiencia internacional y mexicana, realizado por Hijar y Valdez (2008) reveló resultados que indican que las ONG ocupan un lugar privilegiado, en términos de historia y experiencia, en el tema de violencia de género y deben servir de puente entre la sociedad civil y el gobierno. En Latinoamérica se realizan variadas acciones educativas, de consejería y de prevención en la comunidad, pero hay deficiencias en la terapia individual y grupal a las víctimas.

Zabala y Manríquez (2007), en el estudio Caracterización teórico-metodológica de las intervenciones grupales en mujeres victimas de violencia en la pareja, encontraron que las intervenciones más relevantes implicaban utilizar técnicas variadas y eclécticas como las lúdicas, gestálticas, corporales, proyectivas y psicodinámicas. En dichas intervenciones los temas fueron: empoderamiento, toma de decisiones, autonomía, ciclo de violencia, autoestima y mitos. Los beneficios encontrados fueron: superar el aislamiento, generar redes de apoyo, aprender de las demás personas, empoderarse, aumentar la autoestima y reconectarse consigo misma. 


\section{Hipótesis}

H1: Existe una relación entre la ideología de género y la asertividad.

H2: Existe una relación entre la asertividad e ideología de género con el abuso psicológico.

H3: Los talleres de intervención clínica y educativos tendrán un efecto positivo en la ideología de género y en la asertividad de los participantes.

\section{Conclusión}

En conclusión, la base teórica descrita en este artículo enmarca los aspectos principales que intervienen en la experiencia de abuso psicológico, sea sutil o manifiesto, la expresión de una conducta asertiva o no, y, la ideología de género ya sea tradicional o igualitaria. Esta última se incorpora en el proceso de socialización, desde lo más íntimo de la familia hasta otros contextos sociales ampliados como la escuela, la comunidad y la sociedad. Los resultados respecto de la manifestación de abuso psicológico, el tipo de ideología de género predominante y el nivel de asertividad serán expuestos en el próximo artículo.

Este estudio se apoya se apoya sobre la idea de que la experiencia humana es compleja, por lo tanto la comprensión, atención y explicación de la misma implica una visión extensa e integral. Desde ese panorama se intenta describir y vincular estas acepciones a un contexto social concreto, como el ambiente de violencia psicológica que viven muchas mujeres.

\section{Referencias}

Alarcón, R. (2010). El Legado psicológico de Rogelio Díaz-Guerrero. Revista Conducta 16, 1-102. Recuperado de http://www. investigacionesenpsicologia.com.mx/pdf1/ Revista_CONDUCTA-No_16.pdf

Amor, P. J. \& Echeburría, E. (2001). Maltrato psicológico en mujeres víctimas de violencia en el hogar: un estudio comparativo. Revista de Psicopatología y Psicología Clínica 6(3), 167-178. Recuperado de revistas.uned.es

Aranda, M. \& Montes, B. (2012). Las princesas que juegan fútbol y los principes que saltan combas: concientización del alumnado de la presencia de estereotipos y su influencia en la toma de decisiones. Recuperado de https://books.google.com.do/ books/about/Las_princesas_que_juegan_al_f\%C3\%BAtbol_y_lo.html?id=V8J8kwEACAAJ\&redir_esc $=y$

Buesa, S. \& Calvete, E. (2011). Adaptación de la escala de abuso psicológico sutil y manifiesto a las mujeres en una muestra clínica y de la comunidad. Anales de Psicología, 27(3), 774-782. Recuperado de http://revistas.um.es/analesps/ article/download/135391/123581

Calle, S. (2004). Consideraciones sobre la victimización secundaria en la atención social a las víctimas de VG. Revista de trabajo social 4. Recuperado de http://rabida.uhu.es/dspace/handle/10272/180

Cartagena, J. M., Barrero, R. \& Donat, E. (2012). Manual sobre investigación para casos de violencia de pareja y feminicidios en Chile. Fiscalía Nacional Ministerio Público de Chile. Recuperado de http://www.fiscaliadechile.cl/Fiscalia/ archivo?id=3266\&pid=128\&tid $=1 \& d=1$

Castaños, S., Reyes, I., Rivera, S., \& Díaz, R. (2011). Estandarización del inventario de asertividad de Gambrill y Richey-II. RIDEP 1(29). Recuperado de http://www.aidep.org/03_ridep/ R29/r29art2.pdf

Castanyer, O. (1996). La asertividad: expresión de una sana autoestima. Bilbao, España: Editorial Desclee de Brouwer. 
Colombo, G., Ynoub, R., Viglizzo, M., Veneranda, L., Iglesias, G. \& Stopparo, P. (2005). Prevalencia de casos de violencia familiar contra la mujer en la etapa de embarazo, parto y puerperio. Convergencia Revista de ciencias sociales, 38, 81-99. Recuperado de rconvergencia.usemex.mx

Corsi, J. (2003). La violencia en el contexto familiar como problema social. En Corsi, J. (Comp). Maltrato y abuso en el ámbito doméstico: fundamentos teóricos para el estudio de la violencia en las relaciones familiares. Buenos Aires: Paidós.

Culay, A., Santana, F., Rodríguez, R. \& Pérez, C. (2000). Mujer y violencia: ¿un problema de salud comunitario? Revista Cubana de |Medicina General Integral, 16(5), 450-454. Recuperado de http://www.bvs.sld.cu/revistas/mgi/vol16_5_00/ mgi05500.pdf

Davis, S. \& Greenstein, T. (2009). Gender ideology, components, predictor and consequences. Annual Review of Sociology, 35, 87-105. doi: 10.1146/annurev-soc-070308-115920.

Delgado-Álvarez, M., Sánchez-Gómez, M. \& Fernández-Dávila, P. (2012). Atributos y estereotipos de género asociados al ciclo de la violencia contra la mujer. Universitas Psychologica, 11(3), 769777. Recuperado de Base de datos EBSCOhost.

De Lemus, S., Moya, M., Bukowski, M., Lupiáńez, J. (2008). Activación automática de las dimensiones de competencia y sociabilidad en el caso de los estereotipos de género. Psicológica, 29(2), 115-132. Recuperado de http://www.redalyc.org/articulo. oa?id=16929201

Feldman, R. (2007). Desarrollo psicológico a través de la vida. (4ta. Ed.). México: Pearson.

Flores, M. (2010). Etnopsicología de la asertividad. Revista Conducta 16, 1-102. Recuperado de http:// www.investigacionesenpsicologia.com.mx/pdf1/ Revista_CONDUCTA-No_16.pdf
Gaeta, L. \& Galvanovskis, A. (2009). Asertividad: un análisis teórico empírico. Enseñanza e investigación en psicología, 14(2), 403-425. Recuperado de http://www.redalyc.org/articulo. oa?id=29211992013

González, A. \& Castellano, B. (2003). Sexualidad y géneros: alternativas para su educación ante los retos del siglo 21. La Habana: Científico-Técnico.

Hernández, R. \& Pacheco, B. (2009a). Nueva mirada psicológica al "ser mujer", despertar, transgredir y renacer en el arte. Ciencia y Sociedad, 34(3), 331-345. Recuperado de https://revistas.intec.edu. do/index.php/ciso/article/view/Artic3431 Doi: 10.22206/cys.2009.v34i3.pp331-345

Hernández, R. \& Pacheco, B. (2009b). De la culpa a la redención: hacia una nueva psicología. Ciencia y Sociedad, 34(4), 505-515. Recuperado de https://revistas.intec.edu.do/index.php/ciso/article/view/Artic3444 Doi: 10.22206/cys.2009. v34i4.pp505-515

Hijar, M., \& Valdez, R. (2008). Programas de intervención con mujeres víctimas de violencia de pareja y con agresores: experiencia internacional y mexicana. Instituto Nacional de Salud Pública. Recuperado de cedoc.inmujeres.gob.mx

Horno, P. (2009). Amor y Violencia. La dimensión afectiva del maltrato. España: Editorial Descleé.

Instituto Nacional de las Mujeres (2007). El impacto de los estereotipos y los roles de género en México. Recuperado de www.iknowpolitics.org

León, A., Rodríguez, C., Ferrel, R. \& Ceballos, G. (2009). Asertividad y autoestima en estudiantes de primer semestre de la facultad de Ciencias de la Salud en una universidad pública de la ciudad de Santa Marta, Colombia. Psicología del Caribe, 24, 91-105. Recuperado de http://ciruelo.uninorte.edu.co/pdf/psicologia_caribe/24/4_ Asertividad\%20y\%20autoestima.pdf 
Liranzo, P. \& Moreno, L. (2011). Apego y autoestima en estudiantes femeninas de Psicología (tesis de maestría, inédita). Universidad Autónoma de Santo Domingo, República Dominicana.

Ministerio de la Mujer. (s. f.). Manual de procedimientos y protocolos de atención de las casas de acogida o refugios de la República Dominicana. Santo Domingo: Ministerio de la Mujer.

Ministerio de la Mujer. (2012). Ley 24-97 sobre la violencia contra la mujer e intrafamiliar. (12 Ed.). Santo Domingo: Ministerio de la Mujer.

Ministerio de Salud Pública. (2011). Normas nacionales para la atención integral en salud de la violencia intrafamiliar y violencia contra la mujer. Serie de Normas nacionales No. 24. República Dominicana.

Montero, A. (2001). Síndrome de adaptación paradójica a la violencia doméstica: una propuesta teórica. Clínica y Salud, 12(1), 371-397.

Morales J. \& López-Sáez, M. (1996). Creencias estereotípicas sobre la mujer: reflexiones en torno a algunos datos de la situación en España. Recuperado de http://ruc.udc.es/

Moya, M., Expósito, F. \& Padilla, J. L. (2006). Revisión de las propiedades psicométricas de la versión larga y reducida de la Escala de Ideología de Género. International Journal of Clinical and Health Psychology, 6(3), 709-727. Recuperado de http://www.redalyc.org/articulo. oa? id=33760312

O'leary K. D. \& Maiuro, R.D. (Ed.). (2001). Psychological Abuse in Violent Domestic Relations. New York: Springer Publishing Company, Inc.

Organización Mundial de la Salud. (2013). Violencia contra la mujer, violencia de pareja y violencia sexual contra la mujer. Recuperado de www. who.int

Pola, M. J. (2005). El sistema de derecho dominicano y la violencia de pareja. En Candelario, G.
(Comp.). Miradas Desencadenantes: los estudios de género en la República Dominicana al inicio del tercer milenio. Santo Domingo: Centro de Estudios de Género INTEC.

Pick, S., Givaudan, M. \& Prado, A. (2010). Yo quiero, yo puedo prevenir la violencia. Programa breve de sensibilización sobre violencia en el noviazgo. Salud Mental, 33(2), 145-152. Recuperado de scielo.org.mx.

Pourjali, F. \&Zargnaghash, M. (2010). Relationship between assertiveness and the power of saying no with mental health among undergraduate students. Procedia Social and Behavioral Science 9, 137-141. Recuperado de http://www.sciencedirect.com/ science/article/pii/S1877042810022317

Romero, L. (2012). Violencia intrafamiliar: un enfoque socio-cultural. Santo Domingo: Búho.

Vatankhah, H., Daryabari, D., Ghadami, V. \& Naderifar, N. (2013). The effectiveness of communication skills training on self-concept, self-esteem and assertiveness of female students in guidance school in Rasht. Procedia Social and Behavioral Sciences, 84, 885-889. Recuperado de 10.1016/j. sbspro.2013.06.667

Vázquez, F., Torres, A. \& Otero, P. (2010). Prevalencia $\mathrm{y}$ factores de riesgo de la violencia contra la mujer en estudiantes universitarias españolas. Psicothema, 22(2), 196-201. Recuperado de www.unioviedo.es

Velásquez, C., Montgomery, W., Montero, V., Pomalaya, R., Dioses, A., Velásquez, N., Araki, R. \& Reynoso, D. (2008). Bienestar psicológico, asertividad y rendimiento académico en estudiantes universitarios Sanmarquinos. Revista de Investigación en Psicología, 1(2), 139152. Recuperado de http://www.scielo.org.co/ scielo.php?script $=$ sci_nlinks \& ref $=000176 \&$ pi$\mathrm{d}=$ S0120-0534201200030000800050\&lng=en.

Zabala, A. C. \& Manríquez, I. P. (2007). Caracterización teórico-metodológica de las intervenciones 
grupales en mujeres victimas de violencia en la pareja. Universidad de Chile. Recuperado de repositorio. uchile.cl

Zaldívar, D. (2002). El entrenamiento asertivo: manual de instrucción. La Habana: Editorial Féliz Varela.

Zaldívar, D. (1994). La terapia asertiva: una estrategia para su empleo. Revista Cubana de Psicología, 11(1), 53-64. Recuperado de http://pepsic.bvsalud.org/pdf/rcp/v11n1/09.pdf

Zubieta, E., Fernández, I., Vergara, A., Páez, D., Candia, L. \& Martínez, M. D. (1998). Cultura y emoción en América. Boletín de Psicología, 61, 6589. Recuperado de http://www.uned.es/dpto-psicologia-social-y-organizaciones/paginas/profesores/Itziar/Boletin98.pdf

\section{Datos de filiación}

Patricia Liranzo Soto. Es psicóloga clínica, profesora e investigadora del Área de Ciencias Sociales y Humanidades del Instituto Tecnológico de Santo Domingo (INTEC). Posee una maestría en el área de Psicología Clínica por la Universidad Autónoma de Santo Domingo; licenciatura en Psicología; curso Experto en Peritaje Psicológico e Intervención Judicial; curso en Diagnóstico y Rehabilitación Neuropsicológica en Neurología Conductual; curso en Evaluación e Intervención Psicofisiológica en Medicina Conductual. Además se ha desempeñado como facilitadora en OEI-República Dominicana; coordinadora de diplomados y asistente de investigación en el INTEC. Entre sus publicaciones están: Las diosas sometidas: autoconcepto en mujeres de grupos vulnerables. Encuentros afectivo-participativos y sus efectos en el self (2013); Apego y autoestima estudiantes femeninas en Psicología (2012); El proceso de descentralización escolar y sus condiciones de posibilidad (2006). Correo electrónico: patricia.liranzo@ intec.edu.do
Libia Moreno Pérez. Profesora de grado y postgrado de la Escuela de Psicología en la Universidad Autónoma de Santo Domingo (UASD). Tiene maestría y una especialidad en Psicología Clínica por la Universidad Autónoma de Santo Domingo (UASD); postgrado en Educación y Terapia Sexual; Especialidad en Psicoterapia; postgrado en Psicodrama; Entrenamiento en Terapia de Grupo; licenciada en Psicología egresada de la Universidad de la Habana, Cuba. Ha realizado cursos de Psicología de la Salud, Neuropsicología, Violencia de Género, entre otros. Se desempeña como psicóloga clínica en el departamento de Salud Mental de la Maternidad Nuestra Señora de la Altagracia, en Santo Domingo; es además coordinadora de la Maestría en Psicología Escolar en Centro UASDSan Juan de la Maguana.Ha sido expositora en numerosos eventos científicos en la Habana, Panamá, Puerto Rico.

Entre sus publicaciones están Apego y Autoestima en estudiantes femeninas de psicología (2012) y Aplicaciones del Psicodrama en diversas áreas de la psicología (2004).

Correo electrónico: 1mp5012@gmail.com 